\title{
Mechanical eye model for the comparison of optical imaging quality and physiology of human vision
}

\author{
A. Drauschke, E. Rank, S. Auer, M. Forjan and L. Traxler \\ Institute of Biomedical Engineering, University of Applied Sciences Technikum Wien, Vienna, Austria, \\ andreas.drauschke@technikum-wien.at
}

\begin{abstract}
The implantation of intraocular lenses (IOL) is one of the most common surgical interventions. This surgery is performed using different types of IOL, like mono- and multifocal lenses. The measurement and test instructions for IOL are defined in ISO 11979-2[1]. Even though IOL have a high importance there are only approaches for the actual objective evaluation of the quality of an IOL. The reason is given by a still poorly understood human physiology of vision. A direct comparison between physical imaging properties - for example defined by the modulated transfer function (MTF), point spread function (PSF), spot diagrams, Strehl ratio or the coefficients of Seidel- or Zernike polynomials - and physiological perception will allow the development of necessary objective quality standards, also for the development of new intraocular lenses. A mechanic eye model in the scale 1:1 is presented, which has been designed to allow the derivation of the intended quality standards. This is achieved by a simultaneous measurement of physiological and physical imaging properties. Moreover the eye model allows the automatic analysis of the tolerance for tilting and decentrating the IOL perpendicular to the optical axis.
\end{abstract}

\section{Introduction}

Cataracts are the most common reason for the loss of vision for persons with an age over 55. Therefore the cataract surgery is the most common ophthalmologic and one of the most frequent surgeries in general. Estimations take a yearly amount of more than 11 million ambulant cataract surgeries worldwide. In Austria there are approximately 80.000 and in Germany about 700.000 surgeries of this type per year. During this surgery the natural but clouded lens of the human eye is replaced by an artificial intraocular lens (IOL).

There are different types of IOL. Monofocal IOL have distinguished optical imaging properties. Due to the fact that a fixed distance is focused it is inevitable to use additional glasses for focusing on images in the near field. Bifocal IOL have two foci with a larger distance between each other, whereas multifocal IOL often have line foci. Both types allow clear vision in the near field as well as in the far field of the patient's vision. In this case the image in a specific focus is superimposed to the images of the other foci. This effect leads to perceptible ghost images and Halo phenomena, which can be perceived as disturbing by the patients.

Even though the ratio of necessary explantations is very low (about $0.5 \%$ ), the optical imaging properties of the implanted IOL are not always satisfactory for the patients. Therefore more efforts are put into the individualisation of IOL in order to achieve better imaging properties. The classic spherical IOL (sIOL) are increasingly replaced by aberration-free IOL (fIOL), average spherical aberration IOL (aIOL) for the correction of high spherical aberration of the eye's cornea and customized spherical aberrationcorrection IOL (cIOL) [2].
The imaging properties of the IOL may change during the time of usage. A post-operative shift or tilt of the IOL may particularly lead to a decrease of the imaging properties.

Due to the poorly understood physiology of vision, patients might tolerate such artefacts in a differently. Comparing studies dealing with the influence of such postoperative changes of the IOL position can be performed using either numerical eye models [3-5] or mechanical eye models based on actual IOL. [2, 6-11]

Typically the mechanical models are characterised by a complex and open setup on optical working benches. [7-9] In order to be able to define more objective quality parameters it seems necessary to develop a robust, compact, and transportable mechanical eye model, which allows automated series of measurements for tolerance analysis. The aim of this study is the development of such a mechanical eye model.

The geometric and optical properties of the presented eye model are based on the work of Liou \& Brennan [3] which simulates best the anatomical biometry and the optical data of a physiological human eye in our knowledge.

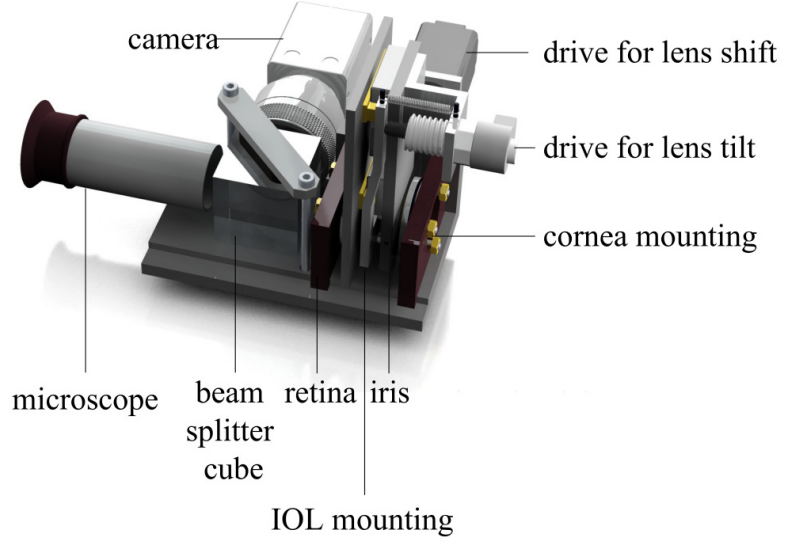

Image 1 General set-up of the mechanical eye model: The most important parts are labelled here 


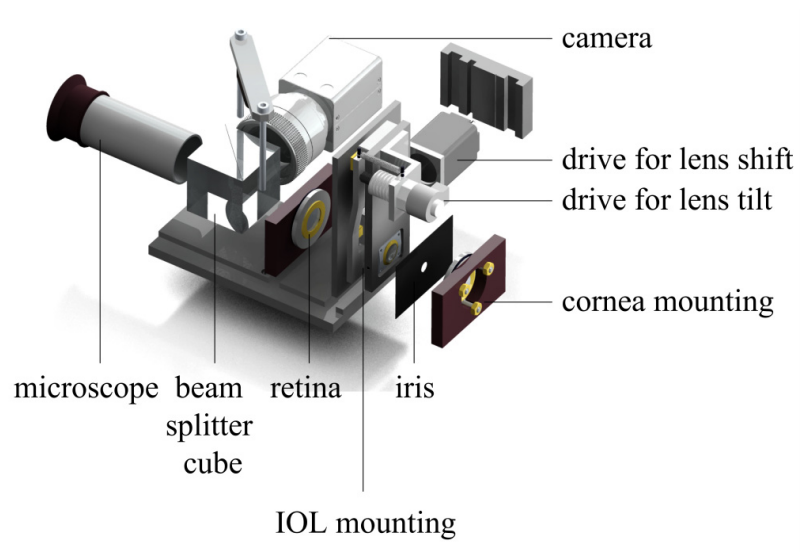

Image 2 General set-up of the mechanical eye model depicted as exploded diagram

\section{Material \& Methods}

\subsection{Mechanical set-up}

Based on the work of Liou \& Brennen [3] the mechanical eye model consists of an interchangeable aspheric convexconcave cornea with high refractivity, a manually changeable aperture diaphragm, simulating the iris, the intraocular lens as test object and a screen as simulation of the retina. The latter has been implemented as plane surface in this eye model. The set-up is depicted in image 1 showing the mounted construction and in image 2 showing the exploded diagram.

The socket of the corneal lens is implemented as lens holder, which allows precise positioning of the lens within

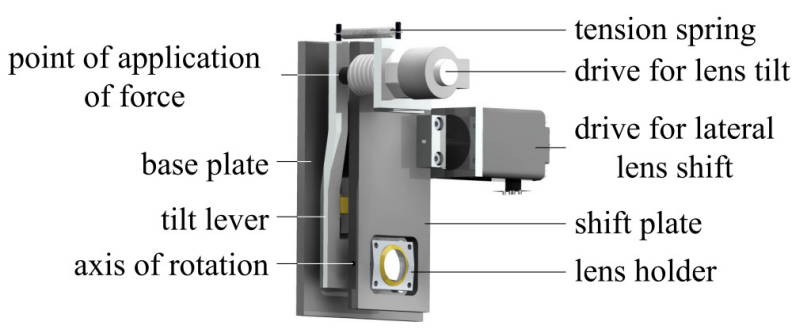

Image 3 Mechanical components for tilt and decentration of the IOL

the optical path. Therefore additional aberrations, which are not correlated to the used IOL, can be eliminated which could occur due to malpositioning of the corneal lens.

The iris has been implemented as thin sheet metal with an aperture centred within the optical path. Moreover the simulated iris can be changed in order to allow the simulation of physiological apertures between $2 \mathrm{~mm}$ to $8 \mathrm{~mm}$. The step size of the simulated iris apertures can be defined by the user.

The IOL is mounted within an individual lens holder. By these means a high flexibility of the set-up is guaranteed, as only the holder has to be replaced in case of an IOL change. The rest of the eye model stays untouched in this case.

The IOL lens holder is mounted on a shift plate. The lateral IOL shift of the entire shift plate (decentration) is performed by a drive stepper motor NEMA $821 \mathrm{~F} 4(\mathrm{X})-\mathrm{V}$. The highest possible accuracy depends on the used threaded rod (defined by $\mathrm{X}$ within the motor labelling) and can be adapted to the needs of the individual user. For the presented eye model the motor type NEMA 8 21F4(AD)-V has been used, which provides a minimal shift of $20 \mu \mathrm{m}$ per step at a maximal speed of $20 \mathrm{~mm} / \mathrm{s}$.

The mentioned shift plate is mounted on a tilt lever. The latter is tilted using the linear stepper motor LC1574W-V. The accuracy of the automatically adjustable tilt is variable over the entire length of the tilt lever. The presented set-up is designed with a distance between the centre of rotation of the tilt lever to the point of application of force of $54 \mathrm{~mm}$ using the stepper motor LC1574W-V with $20 \mu \mathrm{m}$ per step and a maximum shift of (13.35 \pm 0.7$) \mathrm{mm}$ at a maximal velocity of $4 \mathrm{~mm} / \mathrm{s}$. The axis of rotation lies within the optical axis and is positioned $3.2 \mathrm{~mm}$ behind the iris aperture, which means that it is typically not identical to the centre of the IOL. Therefore a tilt causes a vertical shift of the IOL. In order to avoid this vertical shift a concentric centre of rotation has been chosen.

The tilt lever is mounted on a base plate, which can be introduced into the eye model. If other analyses of tolerance shall be performed, like a shift of the IOL parallel to the optical path, the mechanical set-up at the base plate can be changed. This increases the flexibility of the fields of application for the presented eye model.

Due to the fact that tilting and decentering of the IOL can be controlled separately, two-dimensional tolerance analyses can be performed fully automated with this eye model. In order to achieve a high level of integration and usability the same integrated circuit (IC) microstepping driver with translater A3967 by Allegro MicroSystems Inc has been chosen for both motors. These ICs are controlled via the USB interface NI USB 6008 by National Instruments. The graphical user interface (GUI) will be programmed in LabView.

The space between cornea and retina has been designed as wet cell and can therefore be filled with salted water in order to simulate the actual refractive index in front or behind the eye lens. These two refractive indices are identical according to Liou \& Brennan [3]. Therefore a mechanical separation of the two chambers, before and behind the tested IOL, is not necessary for the realisation of different refractive indices.

\subsection{Optical set-up}

Due to the interchangeability of the cornea in the model, different corneal lenses can be used depending on the application case. In order to reach high approximation to an anatomically realistic human cornea, concave-convex aspherical lenses should be used. The human eye is not a perfect imaging system in an optical sense. Therefore the used lens should have a small ration of aspheric aberration. 


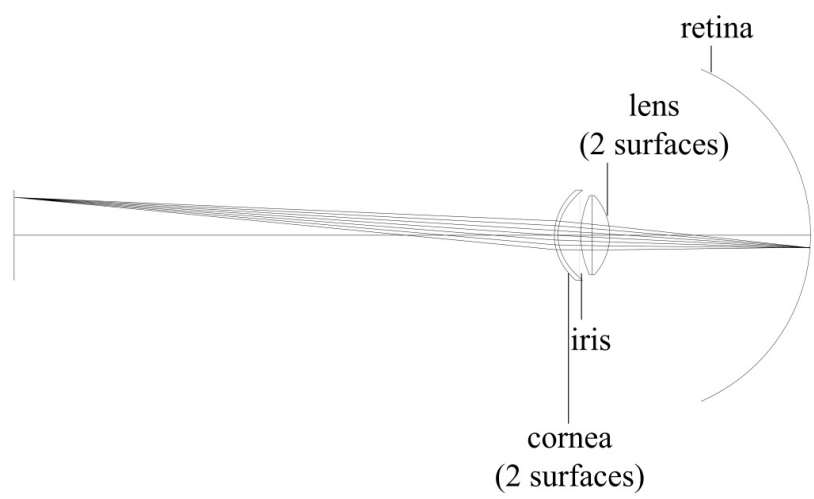

Image 4 Human eye according the model by Liou and Brennan [3]

The latter may vary from patient to patient. The spherical aberration is defined by the radiuses of the lens $R$ and by the cornea and its asphericity $Q$. These values are defined separately for the front and the backside of the cornea in the eye model by Liou \& Brennan.

The values of the simulated cornea for the first plane are $R_{1}=7.77 \mathrm{~mm}$ and $Q_{1}=-0.18$ and for the second plane $R_{2}=$ $6.4 \mathrm{~mm}$ and $Q_{2}=-0.60$, according to image 4 . The focal distance of the cornea in the model based on the work of Liou and Brennan equals $\mathrm{D}=41.85 \mathrm{dpt}$ and is therefore slightly below the value of the most eye models, which would be $43 \mathrm{dpt}$. The front- and the rear focal distance are identical. It is therefore possible to calculate the optical refraction power of the cornea using a thin convexconcave condenser lens with a optical refraction power of $41.85 \mathrm{dpt}$. In order to mechanically simulate a cornea as realistic as possible the mean values of a large amount of samples should not be used. A better solution is to use different corneal lenses with varying values in a range of $D_{\min }$ $=40 \mathrm{dpt}$ to $D_{\max }=47 \mathrm{dpt}$ and $Q_{\min }=-0.44$ to $Q_{\max }=0.03[2]$. Like in the case of natural human lenses, the spherical aberration is corrected partly by the implanted IOL.

In the above mentioned optical model the refractive indices between cornea and lens and between lens and retina equals are 1.336. The lens itself consists of a front part with the values $R_{3}=12.4 \mathrm{~mm}, Q_{3}=-0,94$ and a refractive index of 1.368 and a rear part with values of $R_{4}=-8.1 \mathrm{~mm}$, $Q_{4}=0.96$ and a refractive index of 1.407 . Therefore this lens can understand as a thin lens with a refractive power of $17.78 \mathrm{dpt}$ whereas the entire eye has an optical refractive power of $60.36 \mathrm{dpt}$. It is known that the sum of the optical refractive power of cornea and lens is smaller than the total optical refractive power of the eye. It is important for the practical design of an IOL that, according to the eye model of Liou \& Brennan, the defined gradient of the refractive index of $5.53 \mathrm{dpt}$ adds up to the total optical refractive power. This contribution has to be added via the curvature of the lens using gradient free lenses. In first approximation level the dispersion within the different media can be neglected also in case of illumination with white light.

The eye model can be illuminated with different light sources in the mechanical model. The Strehl ratio can be defined by using an expanded laser illumination. The laser should not be used to determine the physiological image quality. In this case incoherent monochromatic light sources should be used, especially if a high image quality has to be achieved at the camera using a monochromatic camera. An illumination with white light is especially suitable for comparing studies of physiological and physical imaging properties of IOL.

\subsection{Measurement set-up}

The evaluation of the optical quality of the used IOL is performed using an additional set-up, as shown in images 1,2 and 5 , which is attached to the artificial retina. The goal of this set-up is a simultaneous comparison of the physiological perception, which the used IOL causes for the test person, and the photographic recording of the image in vision. In order to achieve the intended simultaneous recording a beam splitter cube is used attached on the rear side of the retina. This set-up then includes a physical section of measurement perpendicular to the optical path of the eye model and a section of measurement of the physiologically perceived image quality by the test person lying parallel to the optical path of the eye model.

The image projected onto the retina can be seen artificially by using a microscope. In the simplest case a 4-f-set-up is used in order to project the image of the artificial retina onto the retina of the test person in a scale of $1: 1$. The objective lens is placed exactly in the distance of its focal length to the artificial lens on that account and is observed via the microscope. In order to assure sharp vision also for ametropic test persons an additional ocular is used, solely correcting the effects of ametropia.

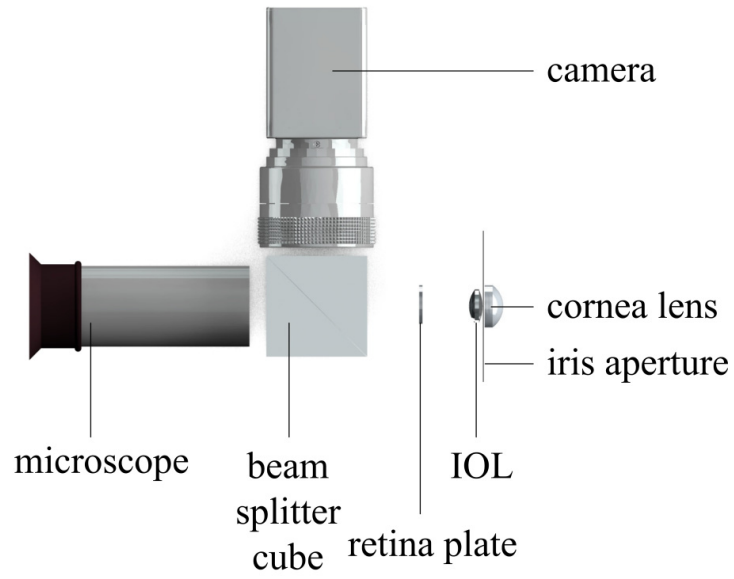

Image 5 Sketch of the measurement set-up including optical components of the eye model

The recording of the images for the physical evaluation of the image properties can be performed either poly- or monochromatically. The achievable image quality is in any case higher when monochromatic systems are used. Nevertheless the hardware which has to be implemented is defined by the field of application.

For polychromatic recordings the NI 1176C smart camera by National Instruments will be used. The physical resolution of the CCD is $1600 \times 1200$ pixels. The camera allows 10 images per second and is combinable with commercially available camera objectives using a C-mount. 
An alternative would be the use of a NI 1778 smart camera by National Instruments. This camera model allows the recording of 15 images per second with a monochromatic resolution of $2448 \times 2050$ pixels. Like described before this camera is also combinable with commercially available objectives via a C-mount.

Depending on the chosen quality property different test patterns can be used for the evaluation of the recorded test images. A standard setting includes USAF-Targets or square or hexapolar spot patterns.

\section{Results}

The presented eye-model has been constructed in the scale of $1: 1$, but still allows the automated and independent tilting and decentering of the implemented IOL.

The described lens holder has been designed in such a way that different lenses can be integrated into the eye-model for the cornea and for the IOL. Therefore the eye-model can be used for both, the definition of new quality standards and the quality check for a new IOL, which might need a new holder.

By using a microstepping driver with the translator A3967 microstepping and as a consequence continuous movements of the mechanic components are guaranteed. The latter characteristic of the system increases the mechanic stability of the entire system and simultaneously decreases the amount of necessary energy, as lower forces are needed. The presented set-up achieves a decentralisation of the IOL with a maximal resolution of $x_{\text {step }}=20 \pm 1 \mu \mathrm{m}$ with a decentering in the range between $x_{\min }=-5 \mathrm{~mm}$ and $x_{\max }=+5 \mathrm{~mm}$. The smallest possible step size for the tilting of the IOL is $\alpha=(1.3 \pm 0.1)^{\prime \prime}$. The tilting is generally possible in a range between $\alpha_{\min }=0^{\circ}$ and $\alpha_{\max }=5^{\circ}$. One automated measurement cycle for $100 \times 100$ points needs 11 minutes and 7 seconds (monocromatic measurement) or 16 minutes and 40 seconds (polychromatic measurement).

By using the smart cameras of National Instruments one integrative graphical user interface can be used for controlling the motors for tilting and decentering the IOL and for data acquisition as well as interpretation of the measured values. This advantage significantly increases the usability of the entire system.

\section{Conclusion}

A mechanical eye model was presented which can be used for semi-automatic measurement of quality criteria for IOL. The operator can choose the cornea and the iris aperture. The tolerance analysis of decentering and tilting of the IOL is done automatically. The MTF and Strehl ratio are measurable actually. In future additional quality functions will be added as PSF or the measurement of the Zernicke coefficients. The presented eye model can be used for definition of new objective quality criteria for IOL or for measurement of a multiplicity of different quality parameters under realistic physiological conditions.
The physiological can be compared with the instantly measured physical image quality due to the use of a beam splitter cube behind the artificial retina.

A re-design of the eye model is necessary in future, which changes the position of the both drives. Because of that the handling of the model will be simplified and the iris aperture plate can be replaced without extracting the full base plane and all parts which are fixed assembled with it.

\section{$5 \quad$ References}

[1] International Organization for Standardization: ISO 11979-2, Ophthalmic implants - Intraocular lenses Part 2: optical properties and test methods, 1999

[2] Pieh, S., Fiala, W., Malz, A. and Stork, W.: In Vitro Strehl ratios with spherical, abberation-free average, and customized spherical aberration-correcting Intraoculas Lenses: Invest Ophthalmol Vis Sci: 50, 1264-1270, 2009

[3] Liou, H.L. and Brennan, N.A.: Anatomically accurate, finite model eye for optical modeling: J. Opt. Soc. Am. A: 14, 1684-1695, 1997

[4] Escudero-Sanz, I. and Navarro, R.: Off-Axis Aberrations of a Wide-Angle Schematic Eye Model: J. Opt. Soc. Am. A: 16, 1881-1891, 1999

[5] Storani de Almeida, M. and Cavalho, L.A.: Different Schematic Eyes and heir Accuracy to the in vivo Eye A Quantitative Comparison Study: Braz. J. of Phys: 37, 378-387, 2007

[6] Norrby, N.E.S.: Standardized methods for assessing the imaging quality of intraocular lenses: Appl. Opt.: 34, 7327-7333, 1995

[7] Norrby, S., Piers, P., Campbell, C., and van der Mooren, M.: Model eyes for evaluation of intraocular lenses: Applied optics: 46, 6595-6605, 2007

[8] Barcik, A., Nowak, J., Siedlecki, D., Zając, M. and Zarówny, J.: Physical model of human eye with implantable intraocular lenses: Proc. of SPIE: 7141, 2008

[9] Pieh, S., Marvan, P., Lackner B., Hanselmayer G., Schmidinger, G., Leitgeb, R., Sticker, M., Hitzenberger, C.K., Fercher, A.F. and Skorpik, C.: Quantitative performance of bifocal and multifocal intraocular lenses in a model eye: point spread function in multifocal intraocular lenses: Archives of Ophthalmology: 120, 23-28, 2001

[10] Gobbi, P.G. Fasce, F., Bozza, S., and Brancato, R.: Experimental characterization of the imaging properties of multifocal intraocular lenses: In Ophthalmic Technologies XIII: Editors: Manns, F., Söderberg, P.G. and Ho, A.: Proceedings of the SPIE: 4951, 104111,2003

[11] Bolognesi, G., Gobbi, P. Fasce, F. Bozza, S. and Brancato, R.: Quality of vision with three different IOLs: A study with the support of an experimental eye model: Invest Ophthalmol Vis Sci: 45, 1385-1396, 2004 